\title{
Adaptación del Cuestionario de Regulación Emocional (ERQ) en población adulta de la Ciudad Autónoma de Buenos Aires y el Conurbano Bonaerense
}

\author{
Alejandro Emilio Pagano ${ }^{1}$ y Nicolás Alejandro Vizioli²
}

\begin{abstract}
RESUMEN
El presente estudio consistió en la adaptación lingüística, conceptual y métrica del Cuestionario de Regulación Emocional (ERQ; Gross \& John, 2003). El mismo es una medida de autoinforme conformada por 10 reactivos que permiten la evaluación de dos estrategias de Regulación Emocional: la reevaluación cognitiva (6 items) y la supresión expresiva (4 reactivos). Para llevar adelante la adaptación se realizó una traducción directa del cuestionario del inglés al español seguido por procedimiento de juicio de expertos y un análisis de discriminación de reactivos. Luego de esto se indagó la estructura factorial de la versión a partir de análisis factoriales confirmatorios en una muestra compuesta por 250 adultos entre 18 y 63 años $(M=32.28, D E=11.74)$, residentes en la Ciudad Autónoma de Buenos Aires y en el Conurbano Bonaerense. Por último, se buscó evidencia de la confiabilidad del instrumento y se establecieron valores normativos. Los resultados indican que la versión adaptada del ERQ presenta adecuada validez de contenido (porcentajes de acuerdo entre $.80 \mathrm{y} 1$ ), adecuada validez factorial para el modelo de dos factores independientes, $\mathrm{S}-\mathrm{B} \chi^{2}(27)=61.98, N N F I=.91, C F I=.93$, RMSEA $(.05-.10)=.07, A I C=7.98$, evidenciando índices de consistencia interna aceptables $(a$ ordinal $=.81$ y $\omega$ ordinal $=.87$ para el factor reevaluación cognitiva; y $a$ ordinal $=.71 \mathrm{y} \omega$ ordinal $=.79$ para el factor de supresión expresiva).
\end{abstract}

\section{Palabras clave: regulación emocional, reevaluación cognitiva, supresión expresiva,} adaptación ERQ.

\section{Adaptation of the Emotional Regulation Questionnaire (ERQ) in adult population of the City of Buenos Aires and the Greater Buenos Aires}

\begin{abstract}
The present study consisted of the linguistic, conceptual, and metric adaptation of the Emotional Regulation Questionnaire (ERQ; Gross \& John, 2003). It is a self-report measure made up of 10 items that allow the evaluation of two Emotional Regulation strategies: cognitive re-evaluation (6 items) and expressive suppression ( 4 items). To carry out the adaptation, a direct translation of the questionnaire from English to Spanish was performed, followed by an expert judgment procedure and a reagent discrimination analysis. Then, a factor structure of this version was analyzed with confirmatory factor analyses in a sample composed of 250 adults between 18 and 63 years $(M=32.28, S D=$ 11.74), residents from Buenos Aires City and Greater Buenos Aires. Finally, evidence of the reliability of the instrument was sought and normative values were established. The results indicate that the adapted version of the ERQ presents adequate content validity (percentages of agreement between .80 and 1), adequate factorial validity for the model of ${ }^{1}$ Universidad de Buenos Aires, Argentina; paganoalejandro@gmail.com; https://orcid.org/0000-0003-4817-
9145

2 Universidad de Buenos Aires, Argentina; nicovizioli@gmail.com; https://orcid.org/ 0000-0002-6113-6847
\end{abstract}


two independent factors $\mathrm{S}-\mathrm{B} \chi^{2}(27)=61.98, N N F I=.91 ; C F I=.93 ;$ RMSEA $(.05-.10)$ $=.07, A I C=7.98$, showing acceptable internal consistency indices (a ordinal $=.81$ and $\omega$ ordinal $=.87$ for the cognitive reappraisal factor; and a ordinal $=.71$ and $\omega$ ordinal $=.79$ for the expressive suppression factor.

Keywords: emotion regulation, cognitive reassessment, expressive suppression, ERQ adaptation.

La regulación emocional ( $\mathrm{RE}$ ) refiere a los procesos a través de los cuales un individuo influye sobre sus emociones, cuando las tiene, cómo las experimenta y expresa (Gross, 1998). Determina con qué facilidad las personas pueden abandonar un estado emocional determinado (Koole, 2009), $\mathrm{y}$ es un proceso de vital relevancia para lograr estados afectivos deseados y metas intencionales (Gross, 2002). Al respecto, en el ámbito clínico, se ha encontrado que estrategias desadaptativas de RE se asocian con un aumento en la sintomatología en distintas patologías (Aldao, Nolen-Hoeksema, \& Schweizer). En este sentido, la $\mathrm{RE}$ ha cobrado gran importancia con la aparición de enfoques psicoterapéuticos transdiagnósticos, que posibilitan clasificar e integrar los diferentes sintomas y diagnósticos desde procesos y factores comunes (Sandin, Chorot, \& Valiente, 2012). Por tanto, existen distintas terapias dirigidas fundamentalmente al trabajo con la RE, como el Protocolo Unificado (Barlow et al., 2010), la Terapia Dialéctico Conductual (Linehan, 1993) o la Terapia de Regulación Emocional (Mennin, 2006).

La RE incluye la manipulación de los antecedentes emocionales de uno mismo y de otros, así como a los componentes fisiológicos, subjetivos o conductuales de las respuestas emocionales (Gross \& Levenson, 1993). Respecto de la manipulación de las respuestas emocionales, pueden distinguirse la RE centrada en el antecedente y la RE centrada en la respuesta (Gross \& Muñoz, 1995). La primera refiere al proceso puesto en funcionamiento por el individuo antes de que comience una emoción y que puede afectar a la propia respuesta emocional. La RE centrada en la respuesta consiste en la manipulación de una respuesta emocional en curso, es decir, una vez que se ha producido.

Teniendo en cuenta esta distinción, Gross (1998) propone diversas formas de $\mathrm{RE}$, tales como la selección de la situación, su modificación, el despliegue atencional, el cambio cognitivo y la modulación de la respuesta emocional. En la elaboración de su modelo de RE, Gross y John (2003), agrupan las diferentes estrategias de $\mathrm{RE}$ en dos grupos: la reevaluación cognitiva y la supresión expresiva. La reevaluación cognitiva implica un cambio a nivel cognitivo y consiste en modelar mentalmente una situación antes de que se desarrolle y considerar sus posibles consecuencias. La supresión expresiva está vinculada a influir sobre una respuesta emocional una vez que esta ha iniciado, e implica la inhibición del comportamiento expresivo del estado emocional. Este modelo es uno de los más difundidos en la literatura investigativa y, para su evaluación, los autores han construido el Cuestionario de Regulación Emocional (ERQ; Gross \& John, 2003).

El ERQ evalúa los dos factores de la RE antes mencionados: la reevaluación cognitiva y la supresión expresiva. Los autores obtuvieron evidencias para sostener esta solución factorial mediante la realización de análisis factoriales exploratorios y confirmatorios. A su vez, reportaron índices 
de consistencia interna adecuados para ambos factores, así como valores adecuados en relación con la confiabilidad test-retest, indicadores de convergencia y de validez discriminante. Hallaron que quienes tienden a utilizar en mayor medida la reevaluación cognitiva por sobre la supresión expresiva experimentaron mayor estado de ánimo positivo, funcionamiento social y bienestar psicológico, así como un menor estado de ánimo negativo y depresivo.

Con relación a adaptaciones realizadas, Balzarotti, John, y Gross (2010) realizaron la adaptación italiana del ERQ en una muestra de 416 adultos estudiantes universitarios. Hallaron que se replicaba la estructura de dos factores, presentando valores adecuados de consistencia interna para ambos. A su vez, reportaron evidencias de validez convergente. Encontraron que la reevaluación cognitiva se asoció positivamente con la reinterpretación positiva, el afecto positivo y la extraversión, y negativamente con el afecto negativo y el neuroticismo. Mientras que la supresión expresiva correlacionó negativamente con la liberación de emociones, el afecto positivo, la extraversión, el apoyo y la diversión social.

En población general, Wiltink et al. (2011) estudiaron las propiedades psicométricas de la versión alemana del ERQ (Abler \& Kessler, 2009) en una muestra de 2524 adultos alemanes. Hallaron la existencia de índices de consistencia interna adecuados. Sin embargo, observaron que dos de los ítems del cuestionario, 8 y 9, correlacionaban con ambos factores. Mediante un análisis factorial confirmatorio, llegaron a la conclusión de que los índices de bondad de ajuste del modelo mejoraban al permitir que el item 8 cargara para ambos factores.

Por su parte, Spaapen, Waters, Brummer, Stopa y Bucks (2014) estudiaron las propiedades psicométricas del ERQ en dos muestras comunitarias de Australia $(n=550)$ y el Reino Unido $(n=483)$, cuyas edades fueron comprendidas entre los 17 y los 95 años. Mediante análisis factorial confirmatorio, hallaron que la estructura factorial del instrumento original no se sostenía en ninguna de las muestras. Sin embargo, hallaron que, descartando el item 1, los indices de bondad de ajuste eran adecuados y que la invarianza era equivalente en ambas muestras y en características demográficas tales como edad, género y educación. A su vez, aportaron evidencia de que el género, la depresión, la ansiedad y el estrés se asociaron significativamente con la reevaluación cognitiva y la supresión expresiva.

A su vez, Rice, Treeby, Gersh, Ogrodniczuk y Kealy (2018) realizaron un estudio psicométrico de la versión de 9 ítems del ERQ publicada por Spaapen et al. (2014), y de la versión original, en 233 adultos australianos y 247 canadienses. Hallaron que la versión de 9 ítems mostró mejores índices de bondad de ajuste que la versión original, ofreciendo indices adecuados $y$ pobres respectivamente.

Con relación al idioma español, Cabello, Salguero, Fernández-Berrocal, \& Gross (2013), adaptaron el instrumento en una muestra de 866 adultos españoles de entre 18 y 80 años. Mediante análisis factoriales confirmatorios, también encontraron que se replicaba la estructura de dos factores esperada, así como índices de consistencia interna adecuados. Aportaron estudios de confiabilidad test-retest y de validez convergente y discriminante. A su vez, informaron que la reevaluación se asoció positivamente con la emoción positiva, mientras que la supresión se asoció negativamente. Por último, la 
reevaluación se asoció positivamente con el funcionamiento social, mientras que la supresión se asoció negativamente.

En Latinoamérica, Gargurevich y Matos (2010) realizaron la validación y adaptación de la versión peruana del ERQ en una muestra de 320 estudiantes. Comprobaron la estructura factorial del instrumento mediante la realización de un análisis factorial exploratorio y uno confirmatorio. De esta manera, hallaron que se replicaba la estructura del instrumento original. A su vez, hallaron índices adecuados de consistencia interna.

En Argentina, se han hallado dos adaptaciones de instrumentos que evalúan la RE desde otros modelos. Por un lado, Medrano, Moretti, Ortiz, y Pereno (2013) realizaron la adaptación del Cuestionario de Regulación Emocional Cognitiva (CERQ; Garnefski, Kraaij, \& Spinhoven, 2001) en una muestra de 359 estudiantes universitarios de instituciones públicas y privadas de las ciudades de Córdoba y Río Cuarto. Este instrumento evalúa nueve dimensiones de la regulación emocional cognitiva: rumiación, catastrofización, auto-culparse, culpar a otros, poner en perspectiva, aceptación, focalización positiva, reinterpretación positiva y refocalización en los planes. A partir de ecuaciones estructurales, hallaron evidencia de una adecuada estructura factorial del instrumento. Reportaron indices de consistencia interna aceptables y evidencias de validez de criterio a partir de correlaciones de los puntajes del CERQ con medidas de Emociones Positivas, Emociones Negativas e Interferencia Emocional.

Por otro lado, Medrano y Trógolo (2014) realizaron la adaptación de la Escala de Dificultades en la Regulación Emocional (DERS; Gratz \& Roemer, 2004) en una muestra de 211 estudiantes universitarios provenientes de distintas carreras de instituciones públicas y privadas de la ciudad de Córdoba. Esta escala evalúa seis factores: dificultades en el control de impulsos, acceso limitado a estrategias de RE, falta de aceptación emocional, interferencia en conductas dirigidas a metas, falta de conciencia emocional, falta de claridad emocional. Mediante análisis factoriales exploratorios, hallaron que la estructura factorial reflejó las dimensiones mencionadas. A su vez, reportaron estudios de consistencia interna y validez concurrente con las escalas de neuroticismo y extraversión del cuestionario IPIP-FFM (Gross, Zalazar Jaime, Piccolo, \& Cupani, 2012), evidenciando resultados satisfactorios. Por último, encontraron diferencias según el género únicamente en el factor falta de claridad emocional en favor de las mujeres.

Los antecedentes mencionados reflejan que el ERQ ha obtenido evidencias de validez de constructo y confiabilidad en adaptaciones realizadas en distintas regiones y poblaciones. Inclusive, se han hallado estudios que indicaron propiedades psicométricas satisfactorias en el idioma español, en España y Perú. Sin embargo, no se ha hallado en Argentina un instrumento que evalué RE desde el modelo propuesto por Gross y John (2003). La adaptación local del ERQ implicaría la posibilidad de medir la reevaluación cognitiva y la supresión expresiva.

Dada la evidencia obtenida en distintas regiones acerca de las propiedades psicométricas del ERQ y la ausencia de una adaptación argentina, el objetivo general de la presente investigación fue realizar la adaptación lingüística, conceptual y métrica del ERQ en adultos de la Ciudad de Buenos Aires y el Conurbano Bonaerense. Los objetivos específicos fueron a) indagar evidencias de validez de contenido; b) aportar evidencias de validez 
de constructo; c) brindar evidencias de la consistencia interna de las puntuaciones; d) establecer valores normativos.

\section{METODOLOGÍA}

\section{Participantes}

Mediante un muestreo no probabilístico de tipo autoelegido (Bologna, 2011) se recolectó una muestra compuesta por 250 participantes, de los cuales 49.2\% $(n=123)$ residian en la Ciudad Autónoma de Buenos Aires y el $50.8 \%(n=127)$ en el Conurbano Bonaerense. Con relación al género, el $40 \%$ $(n=100)$ reportó varón, y el $60 \%(n=150)$ mujer. En cuanto a la edad de los participantes, hubo casos entre 18 y 63 años $(M=32.28, D E=11.74)$. Respecto al nivel de instrucción, el $0.4 \%(n=1)$ manifestó tener el primario incompleto, el $1.2 \%(n=3)$ el secundario incompleto, el $9.6 \%(n=24)$ el secundario completo, el 9.6\% $(n=24)$ terciario incompleto, el $8 \%(n=20)$ terciario completo, el $39.2 \%(n=98)$ universitario incompleto, el $24.4 \%(n=$ $61)$ universitario y el $7.6 \%(n=19)$ posgrado.

La muestra se recogió mediante la difusión del instrumento en redes sociales. La administración se realizó mediante plataformas virtuales. Se procuró que la muestra fuera lo suficientemente grande $(n>200)$ para obtener estimaciones consistentes (Bollen, 1989; Hoyle, 2012) al realizarse análisis factoriales confirmatorios.

\section{Instrumentos}

Para conocer las características sociodemográficas de la muestra, se diseñó un cuestionario que indagó edad, zona de residencia, sexo y nivel de instrucción.

Se realizó la adaptación del Cuestionario de Regulación Emocional (ERQ; Gross \& John, 2003). Este cuestionario autoadministrable consta de 10 items, 6 de los cuales evalúan la reevaluación cognitiva y 4 la supresión expresiva, a los que se responden mediante una escala Likert de 7 puntos, que va de $1=$ totalmente en desacuerdo a $7=$ totalmente de acuerdo. Los autores, mediante análisis factoriales exploratorios y confirmatorios, hallaron que los reactivos se agrupaban en esos dos factores, que exhibieron índices de consistencia interna adecuados, $\mathrm{a}=.79$ para reevaluación cognitiva $\mathrm{y} a=.73$ para supresión expresiva.

\section{Procedimiento}

La recolección de unidades de análisis se realizó mediante la utilización de plataformas virtuales. La administración fue responsabilidad de los autores del presente manuscrito. Se incluyó un consentimiento informado en el cual se especificaron los detalles de los objetivos de la presente investigación junto con las garantías de confidencialidad y anonimato. Se les explicó a los participantes que podian desistir de participar en el momento que lo consideraran.

El consentimiento informado expuso las características de la participación, que fue anónima, voluntaria y sin compensación. Para dar el consentimiento, se incluyeron dos opciones de respuesta al comienzo de la administración que indicaban: "Deseo participar" y "No deseo participar". La 
selección de la primera respuesta permitía completar los instrumentos, mientras que la elección de la segunda finalizaba la administración.

La presente investigación se llevó adelante de acuerdo con la Declaración de Helsinki (World Medical Association, 2013), que establece los principios éticos fundamentales para la investigación con seres humanos.

\section{Análisis de datos}

Se realizaron tres traducciones directas del cuestionario ERQ del inglés al español, siguiendo las recomendaciones de Muñiz, Elosua y Hambleton (2013). Una vez obtenidas las tres versiones en español, se procedió a realizar un juicio de expertos siguiendo las recomendaciones de Escobar-Pérez y Cuervo-Martínez (2008). Los criterios para seleccionar estos jueces fueron: a) experiencia previa en la realización de juicio de expertos; b) experticia en psicometría y evaluación psicológica; c) conocimiento sobre el constructo RE. Una vez seleccionados cinco jueces, se prepararon las instrucciones y planillas para entregárselas, mencionándoles los objetivos del estudio y la consigna respecto al juicio que se esperaba que realizaran. Esta consigna indicaba que el juez, al avanzar en la planilla, se encontraría con un listado de ítems. Al hacerlo, debía valorar las tres traducciones de cada ítem según su grado de claridad, coherencia y relevancia, sobre la base de una escala numérica propuesta. A su vez, se les indicó que cualquier modificación o sugerencia sobre un ítem, así como su propuesta de eliminación, podía ser consignada en una columna de observaciones. Por último, al final de la consigna se les brindó los indicadores necesarios para evaluar cada ítem.

Para evaluar la claridad semántica y sintáctica, los jueces expertos utilizaron una escala likert de cuatro puntos donde 1 indicaba "diferente", 2 "bastante diferente", 3 "bastante similar" y 4 "idéntico", en referencia a si el reactivo se comprendía fácilmente en nuestro contexto cultural. Por otro lado, para evaluar la coherencia de las traducciones, los jueces expertos utilizaron una escala likert de cuatro puntos donde 1 indicaba "no cumple con el criterio", 2 "bajo nivel", 3 "moderado nivel" y 4 "alto nivel". De este modo hacía referencia a si el reactivo tenía lógica respecto a la dimensión o indicador que estaba midiendo. Por último, para evaluar la relevancia de las traducciones, los jueces expertos utilizaron una escala likert de cuatro puntos donde 1 indicaba "no cumple con el criterio", 2 "bajo nivel", 3 "moderado nivel" y 4 "alto nivel". De esta manera hacia referencia a si el reactivo era esencial, muy importante o debia ser excluido.

A través de estas escalas, cada experto respondía leyendo el reactivo de la versión original y luego puntuaba cada una de las tres traducciones, estableciendo la equivalencia semántica, la coherencia y la relevancia de los reactivos. De esta forma, la versión final quedó conformada por los reactivos que mayor puntuación obtuvieron por los jueces. A su vez, se estableció un apartado de observaciones, indicándole al juez que realizara las observaciones respecto a la congruencia del reactivo con la dimensión y aspectos sintácticos que quisiera resaltar (Tornimbeni et al., 2008).

Una vez que se obtuvieron los resultados de cada uno de los jueces, se confeccionó una planilla con todas las valoraciones a partir de la cual se estimó el porcentaje de acuerdo (Tinsley \& Weiss, 1975) y el coeficiente $V$ de Aiken (Aiken, 1985) del juicio realizado por todos los jueces. Estos indicadores de acuerdo están representados por valores que van de 0 a 1: mientras más cercano a 1, el reactivo tendrá mayor validez de contenido. 
Luego de esto, se realizó una prueba piloto, que consistió, según las recomendaciones de Muñiz et al. (2013), en una serie de entrevistas semidirigidas donde se administró el cuestionario y se indagó la comprensión de los reactivos, el tiempo necesario para completarlo y se examinó la posible presencia de errores de contenido y de formato.

Una vez finalizados los procesos antes mencionados, sobre la muestra final $(n=250)$ se realizó un análisis de discriminación de los reactivos (Hogan, 2004). Para esto se utilizó la comparación interna entre extremos (Muñiz, 2005), dividiendo la muestra en cuartiles respecto a la puntuación total obtenida en cada uno de los dos factores de RE. Luego, se comparó mediante el estadístico $U$ de Mann Whitney, debido a que los datos no cumplieron supuesto de normalidad, el cuartil 1 y el cuartil 4 respecto a los valores en cada uno de los ítems.

Para evaluar la validez factorial del ERQ se realizaron análisis factoriales confirmatorios mediante el software EQS 6.1. Al obtenerse una muestra lo suficientemente grande $(n>200)$ para realizar estimaciones consistentes, se utilizó el procedimiento reportado en los estudios de adaptación y validación realizados por Balzarotti et al. (2010), Cabello et al. (2013) y Gross y John (2003). Por lo que se compararon dos modelos: uno menos parsimonioso, en el cual las correlaciones entre dos factores (supresión expresiva y reevaluación cognitiva) se estimaron libremente, y otro independiente, en el cual las correlaciones entre factores se fijaron en el valor 0 .

Al tratarse de datos que no cumplian con los criterios de normalidad, se recurrió al método de estimación de máxima verosimilitud robusto, para la obtención de estadísticos. Este procedimiento posibilita que los resultados no sufran alteraciones significativas, aun cuando no se cumpla el supuesto de normalidad (Manzano Patiño \& Zamora Muñoz, 2009).

Se consideraron los siguientes indices de bondad de ajuste: $\chi^{2}$ de Satorra- Bentler, dividido por los grados de libertad (valores $\leq 5.0$ indican un buen ajuste), de acuerdo con las recomendaciones de $\mathrm{Hu}$, Bentler y Kano (1992); NNFI (Non-Normed Fit Index); CFI (Comparative Fit Index), RMSEA (Root Mean Square Error of Approximation) y SRMR (Standarized Root MeanSquare Residual). De acuerdo con los criterios especificados por Kline (2010) y Schumacker y Lomax (2016), se considera un ajuste aceptable valores de .90 en NNFI y CFI, asi como valores menores o iguales a .08 en RMSEA (Browne \& Cudeck, 1992) y menores a 1 en el SRMR (Schweizer, 2010). A su vez, se tuvo en cuenta el AIC (Akaike's Information Criterion), que arroja valores relativos, de manera tal que se considera el mejor modelo aquel que obtenga un AIC más bajo (Byrne, 2013). La validez de constructo se evaluó a través del examen de las cargas factoriales y de las correlaciones entre los factores. En el primer caso, se consideraron aceptables cargas estandarizadas mayores al límite de .30 (Hair, Black, Babin, Anderson, \& Tatham, 2006; Nunnally \& Bernstein, 1994), en el segundo <.80 (Brown, 2006). En el caso de las correlaciones entre factores, se consideraron los valores $>.19$ como muy bajas, entre $>.20 \mathrm{y}<.39$ como bajas, entre $>.40 \mathrm{y}<.59$ como moderadas, entre $>.60 \mathrm{y}<.79$ como altas $\mathrm{y}<.80$ como muy altas (Brown, 2006; Evans, 1996).

Para evaluar la consistencia interna se calcularon los índices de fiabilidad alfa ordinal y omega ordinal (McDonald, 1999) del inventario. E1 cálculo de índices ordinales se sustenta en que su utilización se recomienda al trabajar con matrices con items ordinales, o con muestras con una distribución no normal y cuando las distribuciones univariadas de elementos 
ordinales son asimétricas o muestran curtosis excesiva (Bryant \& Satorra, 2012). Siguiendo este procedimiento, estos cálculos se realizaron a partir de matrices de correlaciones policóricas. Para ello se empleó el programa $\mathrm{R}$ versión 3.6.0 y los siguientes paquetes de R: GPArotation (Bernaards \& Jennrich, 2005), psych (Revelle, 2018) y Rcmdr (Fox \& Bouchet-Valat, 2019). A su vez, se calcularon las correlaciones corregidas ítem-factor, considerando como adecuados valores superiores a .40. (Nunnally \& Bernstein, 1994). Los indices de confiabilidad se interpretaron siguiendo el criterio de George y Mallery (2003): puntuaciones mayores a $\geq .9$ se consideraron excelentes, $\geq .8$ buenas, $\geq .7$ aceptables, $\geq .6$ cuestionables, $\geq .5$ malas $\mathrm{e} \leq .5$ inaceptables.

Por último, para la obtención de valores normativos, en primer lugar, se analizó la existencia de diferencias según sexo para los factores evaluados por el ERQ, mediante la prueba t de Student. Antes de realizar dicha prueba, se examinaron los gráficos Q-Q para determinar la distribución normal de las puntuaciones. En segundo lugar, se calcularon los puntajes percentilares de los factores mediante el software estadístico SPSS versión 26.

\section{RESULTADOS}

Sobre la base del juicio de expertos, se seleccionaron las 10 traducciones más adecuadas, junto con la escala Likert, donde los jueces sugirieron a partir de los indices de porcentaje de acuerdo y $\mathrm{V}$ de Aiken que una escala de "totalmente en desacuerdo" a "totalmente de acuerdo" representaba conceptualmente la versión en inglés "Strongly disagree" a "Strongly agree". Respecto a los tres criterios establecidos, los ítems seleccionados obtuvieron valores adecuados de porcentajes de acuerdo, entre .80 y 1 (ver tabla 1). Como así también, los coeficientes $\mathrm{V}$ de Aiken oscilaron entre valores de .80 a 1 en todos los casos, valores aceptables según la literatura (Hyrkäs, AppelqvistSchmidlechner, \& Oksa, 2003; Voutilainen \& Liukkonen, 1995).

Tabla 1.

Porcentaje de Acuerdo y V de Aiken del Juicio de Expertos.

\begin{tabular}{lcccccc}
\hline & \multicolumn{2}{c}{ Claridad } & \multicolumn{2}{c}{ Coherencia } & \multicolumn{2}{c}{ Relevancia } \\
\cline { 2 - 7 } & \% Acuerdo & V Aiken & \% Acuerdo & V Aiken & \% Acuerdo & V Aiken \\
\hline EA1 $^{\mathrm{a}}$ & 80 & .93 & 80 & .93 & 80 & .93 \\
EB1 $^{\mathrm{b}}$ & 100 & 1.00 & 100 & 1.00 & 100 & 1.00 \\
EC1 $^{\mathrm{c}}$ & 100 & 1.00 & 100 & 1.00 & 100 & 1.00 \\
ERQ 1 $^{\mathrm{d}}$ & 100 & 1.00 & 100 & 1.00 & 100 & 1.00 \\
ERQ 2 $^{\mathrm{d}}$ & 80 & .93 & 100 & 1.00 & 80 & .87 \\
ERQ 3 $^{\mathrm{d}}$ & 100 & 1.00 & 100 & 1.00 & 100 & 1.00 \\
ERQ 4 & 80 & .87 & 100 & 1.00 & 80 & .80 \\
ERQ 5 & 80 & 0,80 & 100 & 1.00 & 80 & .87 \\
ERQ 6 & 100 & 1.00 & 100 & 1.00 & 100 & 1.00 \\
ERQ 7 $^{\mathrm{d}}$ & 100 & 1.00 & 80 & .93 & 80 & .93 \\
ERQ 8 $^{\mathrm{d}}$ & 80 & .87 & 80 & .87 & 80 & .93 \\
ERQ 9 $^{\mathrm{d}}$ & 100 & 1.00 & 100 & 1.00 & 100 & 1.00 \\
ERQ 10 $^{\mathrm{d}}$ & 80 & .87 & 80 & .93 & 100 & .93 \\
\hline
\end{tabular}

Sin embargo, se realizaron modificaciones en los items 4, 5, 8 y 9 sobre la base de las observaciones brindadas por los jueces. En el item 4 "When I am feeling positive emotions, I am careful not to express them", su traducción fue 
"Cuando estoy sintiendo emociones positivas, tengo cuidado de no expresarlas". A partir de las observaciones, se decidió modificar la expresión del siguiente modo "Cuando estoy sintiendo emociones positivas, me cuido de no expresarlas". En lo que refiere al item 5 "When I'm faced with a stressful situation, I make myself think about it in a way that helps me stay calm", su traducción fue "Cuando me encuentro en una situación estresante, trato de pensar en ella de un modo que me permita permanecer en calma". Se decidió modificar el verbo "permanecer" por "estar", y su traducción final resultó "Cuando me encuentro en una situación estresante, trato de pensar en ella de un modo que me permita estar en calma". Por otro lado, en el ítem 8, "I control my emotions by changing the way I think about the situation I'm in", se realizó una modificación pequeña agregando la palabra "la" al final del reactivo. La versión final fue "Controlo mis emociones cambiando la forma de pensar acerca de la situación en la que estoy". Por último, en el item 9, "When I am feeling negative emotions, I make sure not to express them", se modificó el "aseguro" por "cuido". La versión final resultó "Cuando siento emociones negativas, me cuido de no expresarlas" a partir de las sugerencias y observaciones realizadas por los jueces.

A su vez, en la prueba piloto realizada se consultó sobre estas modificaciones que surgieron a partir del juicio de experto. En todos los casos, los individuos mencionaron comprender de mejor forma las versiones modificadas tras las observaciones de los jueces. De la misma forma, se corrigieron acentos y comas en los items 7 y 10, mientras que el tiempo promedio que emplearon los evaluados para completar el cuestionario fue de 4.20 minutos.

Una vez preparada la versión definitiva, se procedió a aplicar el cuestionario a la muestra utilizada en la investigación. En la tabla 2 se presenta el análisis de los ítems a partir de la comparación entre extremos. Se puede observar que los resultados en la comparación entre cuartil 1 y el cuartil 4 en las dos dimensiones del cuestionario arroja diferencias significativas en todos los casos $p<.01(a=.01)$. Esto indicaría que todos ellos discriminan de forma adecuada.

Para analizar la validez de constructo, se realizó un análisis de las cargas estandarizadas de los ítems considerando el modelo de dos factores independientes. Se hallaron indices de bondad de ajuste adecuados: S-B $\chi^{2}$ $(35)=76.77 ; N N F I=.88 ; C F I=.91 ; S R M R=.07 ;$ RMSEA $(0.06-1.00)=0.08$. Las cargas estandarizadas fueron mayores a .30 en todos los casos, excepto en el item 4 "Cuando estoy sintiendo emociones positivas, me cuido de no expresarlas". Se procedió a descartar este ítem y a continuación se compararon dos modelos, para estudiar la validez factorial del ERQ: uno menos parsimonioso, en el cual las correlaciones entre factores se estimaron libremente, y un modelo de factores independientes, en el cual las correlaciones entre factores se fijaron en 0 . 
Tabla 2

Método Interno de Comparación entre Extremos.

\begin{tabular}{|c|c|c|c|c|c|c|c|c|}
\hline & \multicolumn{2}{|c|}{$\begin{array}{c}\text { Muestra total } \\
\qquad n=250\end{array}$} & \multicolumn{2}{|c|}{ Q1 } & \multicolumn{2}{|c|}{ Q4 } & \multicolumn{2}{|c|}{$\begin{array}{l}\text { U de Mann } \\
\text { Whitney }\end{array}$} \\
\hline & $M$ & $D E$ & $M$ & $D E$ & $M$ & $D E$ & $Z$ & $p$ \\
\hline \multicolumn{9}{|l|}{$\mathrm{RC}$} \\
\hline Ítem 1 & 4.56 & 1.77 & 2.94 & 1.38 & 5.98 & 1.41 & -8.50 & $.000^{*}$ \\
\hline Ítem 3 & 4.71 & 1.79 & 3.14 & 1.71 & 6.19 & 1.01 & -8.46 & $.000^{*}$ \\
\hline Ítem 5 & 4.81 & 1.66 & 3.80 & 1.60 & 6.05 & 1.21 & -7.19 & $.000^{*}$ \\
\hline Ítem 7 & 4.79 & 1.59 & 3.14 & 1.31 & 6.38 & 0.79 & -9.60 & $.000^{*}$ \\
\hline Ítem 8 & 4.59 & 1.59 & 3.05 & 1.36 & 6.00 & 0.96 & -9.00 & $.000^{*}$ \\
\hline Ítem 10 & 4.81 & 1.58 & 3.32 & 1.44 & 6.19 & 1.10 & -8.67 & $.000^{*}$ \\
\hline \multicolumn{9}{|l|}{ SE } \\
\hline Ítem 2 & 3.90 & 1.85 & 1.93 & 1.03 & 5.68 & 1.23 & -9.40 & $.000^{*}$ \\
\hline Ítem 4 & 2.20 & 1.51 & 1.31 & 0.65 & 3.22 & 1.84 & -6.57 & $.000^{*}$ \\
\hline Ítem 6 & 3.12 & 1.85 & 1.20 & 0.41 & 5.24 & 1.09 & -9.91 & $.000^{*}$ \\
\hline Ítem 9 & 3.68 & 1.89 & 1.69 & 0.86 & 5.22 & 1.60 & -8.96 & $.000^{*}$ \\
\hline
\end{tabular}

En relación con el primer modelo, se obtuvieron los siguientes índices de bondad de ajuste: $\mathrm{S}-\mathrm{B} \chi^{2}(26)=61.36, N N F I=.91, C F I=.93$, RMSEA $(.05-.10)=$ $.07, A I C=9.35$. Respecto del modelo de 2 factores independientes, los índices de bondad de ajuste fueron los siguientes: $\mathrm{S}-\mathrm{B} \chi^{2}(27)=61.98, N N F I=.91, C F I=$ .93 , RMSEA (.05-.10) = .07, AIC $=7.98$. Si bien los dos modelos exhiben un ajuste adecuado, el modelo de factores independientes se considera el más adecuado, exhibiendo un AIC más pequeño. En la tabla 3 se informan las cargas factoriales estandarizadas. A continuación, se analizó la existencia de correlaciones entre los factores para confirmar que el instrumento no fuera unidimensional. Se obtuvo una correlación entre factores de $r=.02$, muy baja, confirmando la existencia de dos factores bien diferenciados.

En relación con la consistencia interna del cuestionario, se obtuvieron valores de alfa ordinal $=.81 \mathrm{y}$ de omega ordinal $=.87$ para el factor reevaluación cognitiva; $\mathrm{y}$ alfa ordinal $=.71 \mathrm{y}$ omega ordinal $=.79$ para el factor supresión expresiva. Respecto de las correlaciones corregidas ítem-factor, se obtuvieron valores adecuados en todos los casos. En la tabla 3 se presentan las correlaciones corregidas item-factor.

Para calcular los valores normativos, en primer lugar, se analizó la existencia de diferencias según sexo en los dos factores evaluados por el instrumento. Para ello, se examinaron los gráficos Q-Q para determinar la distribución normal de las puntuaciones. Al cumplirse el supuesto de normalidad, se realizó la prueba $\mathrm{t}$ de Student. Se hallaron diferencias significativas tanto en la reevaluación cognitiva $(p=.02)$ como en la supresión expresiva $(p<.001)$. En el primer caso en favor de las mujeres $\mathrm{y}$, en el segundo, en favor de los varones. Por esta razón, en segundo lugar, se procedió a calcular puntajes percentilares para varones y mujeres por separado (tabla 4). 
Tabla 3.

Cargas factoriales estandarizadas y correlaciones corregidas ítem-factor de los ítems del ERQ.

Ítem

$\mathrm{RC}(\alpha$ ordinal $=0,81 ; \omega$ ordinal $=0,87$ )

1. Cuando quiero sentir más emociones positivas (como alegría o diversión), cambio lo que estoy pensando.

.50

.59

3. Cuando quiero sentir menos emociones negativas (como tristeza o enojo), cambio lo que estoy pensando.

.48

5. Cuando me encuentro en una situación estresante, trato de pensar en ella de un modo que me permita estar en calma.

7. Cuando quiero sentir una emoción más positiva, cambio la manera en que estoy pensando sobre la situación.

8. Controlo mis emociones cambiando la forma de pensar acerca de la situación en la que estoy.

10. Cuando quiero sentir una emoción menos negativa, cambio la manera en que estoy pensando sobre la situación.

SE $(\alpha$ ordinal $=0,72 ; \omega$ ordinal $=0,79)$

2. Me guardo mis emociones para mí mismo/a.

6. Controlo mis emociones no expresándolas

9. Cuando siento emociones negativas, me cuido de no expresarlas.

Nota. $\mathrm{RC}=$ reevaluación cognitiva; $\mathrm{SE}=$ supresión expresiva; $\alpha=$ alfa ordinal; $\omega=$ omega ordinal

Tabla 4.

Puntuaciones normativas de los factores del ERQ.

\begin{tabular}{|c|c|c|c|c|c|}
\hline & \multicolumn{2}{|c|}{ Varones } & \multicolumn{2}{|c|}{ Mujeres } \\
\hline & & $\begin{array}{c}\text { Reevaluación } \\
\text { Cognitiva }\end{array}$ & $\begin{array}{l}\text { Supresión } \\
\text { Expresiva }\end{array}$ & $\begin{array}{c}\text { Reevaluación } \\
\text { Cognitiva }\end{array}$ & $\begin{array}{l}\text { Supresión } \\
\text { Expresiva }\end{array}$ \\
\hline$M$ & & 26.98 & 12.07 & 29.13 & 9.77 \\
\hline$D E$ & & 6.51 & 3.90 & 7.15 & 4.65 \\
\hline Percentiles & 5 & 15 & 5 & 15 & 3 \\
\hline & 10 & 19 & 7 & 19 & 4 \\
\hline & 15 & 20 & 8 & 22 & 4 \\
\hline & 20 & 21 & 8 & 24 & 5 \\
\hline & 25 & 23 & 9 & 26 & 6 \\
\hline & 30 & 24 & 10 & 27 & 6 \\
\hline & 35 & 25 & 11 & 27 & 7 \\
\hline & 40 & 25 & 11 & 28 & 8 \\
\hline & 45 & 26 & 12 & 28 & 9 \\
\hline & 50 & 27 & 12 & 29 & 9 \\
\hline & 55 & 27 & 12 & 29 & 10 \\
\hline & 60 & 29 & 13 & 31 & 11 \\
\hline & 65 & 30 & 14 & 33 & 12 \\
\hline & 70 & 31 & 14 & 34 & 12 \\
\hline & 75 & 32 & 16 & 35 & 13 \\
\hline & 80 & 33 & 16 & 36 & 14 \\
\hline & 85 & 34 & 16 & 36 & 15 \\
\hline & 90 & 36 & 17 & 39 & 17 \\
\hline & 95 & 36 & 18 & 39 & 18 \\
\hline & 100 & 42 & 20 & 42 & 21 \\
\hline
\end{tabular}




\section{DISCUSIÓN}

Con el objetivo de hallar una adaptación con equivalencia lingüística, conceptual y métrica del ERQ en adultos de la Ciudad de Buenos Aires y el Conurbano Bonaerense, en primer lugar, se procedió a realizar una traducción directa del cuestionario del inglés al español, sometiendo tres versiones adaptadas a un juicio de experto. Los resultados del juicio realizado aportan evidencias de validez de contenido por parte de los 9 reactivos que componen la versión final del test. A su vez, la adaptación aporta evidencia del poder de discriminación de los reactivos.

A continuación, se procedió a analizar las propiedades psicométricas de la traducción del cuestionario. La literatura investigativa evidencia que la $\mathrm{RE}$ está compuesta por dos factores -reevaluación cognitiva y supresión expresiva- (Balzarotti et al., 2010; Cabello et al., 2013; Gargurevich \& Matos, 2010; Gross \& John, 2003; Spaapen et al., 2014; Wiltink et al., 2011). De manera congruente, se ha hallado que el modelo de dos factores independientes es el más adecuado. Estos resultados, a su vez, son coincidentes con la teoría de $\mathrm{RE}$, a partir de la cual la reevaluación cognitiva está centrada como antecedente a la respuesta emocional, y consiste en un cambio cognitivo y modelar mentalmente una situación y sus consecuencias. Por otro lado, la supresión expresiva se presenta como independiente, y centrada en la inhibición o manipulación de una respuesta emocional ya iniciada (Gross \& John, 2003).

Se debe señalar que se ha decidido descartar el ítem 4 "Cuando estoy sintiendo emociones positivas, me cuido de no expresarlas" ya que presenta una carga factorial por debajo del criterio establecido. Investigaciones halladas (Rice et al., 2018; Spaapen et al., 2014; Wiltink et al., 2011) presentaron dificultades para conservar los 10 ítems de la versión original. Se considera aceptable la solución final conformada por un total de 9 reactivos, respetando el criterio mínimo de 3 items por factor (Fabrigar, Wegener, MacCallum, \& Strahan, 1999; Ferrando \& Anguiano-Carrasco, 2010). A modo de hipótesis, es posible que la baja carga factorial del reactivo pueda deberse a factores propios de la cultura argentina, en la cual no se llegue a comprender el propósito que puede tener el cuidarse de no expresar emociones positivas y esto sea considerado socialmente mal visto. Se deberian hacer estudios donde se indague sobre esta particularidad que presenta el item 4.

Respecto de la consistencia interna, se obtuvieron valores similares a los hallados en la versión original (Gross \& John, 2003), considerados aceptables y buenos, para los dos factores. Cabe destacar que se calcularon indices ordinales de consistencia interna, dado que, al trabajar con items politómicos, se recomienda la realización de análisis de matrices de correlaciones policóricas (Ferrando \& Lorenzo-Seva, 2014) para evitar posibles infraestimaciones (Gadermann, Guhn, \& Zumbo, 2012). De todas maneras, tratándose de un instrumento con una cantidad reducida de items, no se corre el peligro de aumentar los valores de consistencia interna debido únicamente al resultado del aumento de reactivos (Hair, Black, Babin, \& Anderson, 2010). En este sentido, se ha señalado que instrumentos con más de 20 items pueden tener indices de consistencia interna adecuados, aun cuando las correlaciones entre reactivos fueran pequeñas (Cortina, 1993).

En relación con las puntuaciones normativas, se procedió a calcular puntajes percentilares diferenciados según sexo, habiéndose encontrado la existencia de diferencias significativas en la reevaluación cognitiva en favor de 
las mujeres, y en la supresión expresiva en favor de los varones. Estos resultados son congruentes con los de las investigaciones realizadas por Spaapen et al. (2014). A su vez, coinciden parcialmente con los reportados por Cabello et al. (2013) y Gross y John (2003), quienes reportaron que los varones obtuvieron puntajes mayores de supresión expresiva, pero no hallaron diferencias significativas en la reevaluación cognitiva.

Para concluir, se han obtenido evidencias que permiten establecer que el ERQ es un instrumento válido y confiable, de gran utilidad para su utilización en población de la Ciudad de Buenos Aires y el Conurbano Bonaerense. Se trata de un cuestionario breve y de fácil utilización, que aporta información relevante acerca de las estrategias de RE.

Entre las limitaciones del presente estudio, puede señalarse el empleo de un muestreo intencional no probabilístico, el estudio de la estructura factorial únicamente en población general y el hecho de no haberse realizado estudios sobre la validez convergente o discriminante del instrumento. Se sugiere que futuras investigaciones utilicen muestreos probabilisticos, examinen la estructura factorial en distintas poblaciones y realicen estudios de validez convergente y discriminante. De este modo, se podrá obtener mayor evidencia sobre las propiedades psicométricas del ERQ.

Por último, debido a que la adaptación del instrumento ha sido realizada en población general, se sugiere interpretar prudentemente los resultados cuando se utilice el instrumento en el ámbito clínico.

\section{REFERENCIAS}

Abler, B. \& Kessler, H. (2009). Emotion Regulation Questionnaire - A German version of the ERQ by Gross and John. Diagnostica, 55, 144 -152. https://doi.org/10.1026/0012-1924.55.3.144

Aiken, L. R. (1985). Three coefficients for analyzing the reliability and validity of ratings. Educational and Psychological Measurement, 45(1), 131-142. https://doi.org/10.1177/0013164485451012

Aldao, A., Nolen-Hoeksema, S., \& Schweizer, S. (2010). Emotion-regulation strategies across psychopathology: A meta-analytic review. Clinical Psychology Review, 30(2), 217-237. https://doi.org/10.1016/j.cpr.2009.11.004

Balzarotti, S., John, O. P., \& Gross, J. J. (2010). An Italian adaptation of the emotion regulation questionnaire. European Journal of Psychological Assessment, 26, 61-67. https://doi.org/10.1027/1015-5759/a000009

Barlow, D. H., Ellard, K. K., Fairholme, C. P., Farchione, T. J., Boisseau, C. L., Allen, L. B., \& EhrenreichMay J. T. (2010). Unified protocol for transdiagnostic treatment of emotional disorders: Workbook. New York: Oxford University Press. https://doi.org/10.1093/med:psych/9780199772667.001.0001

Bernaards, C. A. \& Jennrich, R. I. (2005). Gradient Projection Algorithms and Software for Arbitrary Rotation Criteria in Factor Analysis. Educational and Psychological Measurement, 65, 676-696. https://doi.org/10.1177/0013164404272507

Bollen, K. A. (1989). Structural Equations with Latent Variables. Nueva York: John Wiley. https://doi.org/10.1002/9781118619179

Bologna, E. (2011). Estadística para Psicología y Educación. Córdoba: Editorial Brujas.

Brown, T. A. (2006). Confirmatory factor analysis for applied research. Nueva York: Guilford Press. https://doi.org/10.1080/00036810600603377

Browne, M.W. \& Cudeck, R. (1992). Alternative Ways of Assessing Model Fit. Sociological Methods \& Research, 21(2), 8-13. https://doi.org/10.1177/0049124192021002005

Bryant, F. B. \& Satorra, A. (2012). Principles and Practice of Scaled Difference Chi-Square Testing. Structural Equation Modeling: A Multidisciplinary Journal. 19(3), 372-398. https://doi.org/10.1080/10705511.2012.687671

Byrne, B. M. (2013). Structural Equation Modeling with LISREL, PRELIS, and SIMPLIS: Basic concepts, applications, and programming. New Jersey: Lawrence Erlbaum Associates. https://doi.org/10.4324/9780203774762

Cabello, R., Salguero, J. M., Fernández-Berrocal, P., \& Gross, J. J. (2013). A Spanish Adaptation of the Emotion Regulation Questionnaire. European Journal of Psychological Assessment, 29(4), 234-240. https://doi.org/10.1027/1015-5759/a000150

Cortina, J. M. (1993). What is coefficient alpha? An examination of theory and applications. Journal of Applied Psychology, 78(1), 98-104. https://doi.org/10.1037/0021-9010.78.1.98

Escobar-Pérez, J. \& Cuervo-Martínez, Á. (2008). Validez de contenido y juicio de expertos: una aproximación a su utilización. Avances en medición, 6(1), 27-36.

Evans, J. D. (1996). Straightforward statistics for the behavioral sciences. Pacific Grove: Brooks/Cole 
Publishing.

Fabrigar, L. R., Wegener, D. T., MacCallum, R. C., \& Strahan, E. J. (1999). Evaluating the use of exploratory factor analysis in psychological research. Psychological methods, 4(3), 272-299. https://doi.org/10.1037/1082-989X.4.3.272

Ferrando, P. J. \& Anguiano-Carrasco, C. (2010). El análisis factorial como técnica de investigación en psicología. Papeles del psicólogo, 31(1), 18-33.

Ferrando, P. J. \& Lorenzo-Seva, U. (2014). El análisis factorial exploratorio de los ítems: algunas consideraciones adicionales. Anales De Psicología, 30(3), 1170-1175. https://doi.org/10.6018/analesps.30.3.199991

Fox, J., \& Bouchet-Valat, M. (2019). Rcmdr: R Commander. R package version 2.5-2.

Gadermann, A. M., Guhn, M., \& Zumbo, B. D. (2012). Estimating ordinal reliability for Likert-type and ordinal item response data: A conceptual, empirical, and practical guide. Practical Assessment, Research \& Evaluation, 17, 1-13.

Garnefski, N., Kraaij, V., \& Spinhoven, P. (2001). Negative life events, cognitive emotion regulation and emotional problems. Personality and Individual Differences, 30(8), 1311-1327. https://doi.org/10.1016/S0191-8869(00)00113-6

Gargurevich, R. \& Matos, L. (2010). Propiedades psicométricas del cuestionario de autoregulación emocional adaptado para el Perú (ERQP). Revista de Psicología, 12(1), 192-215.

George, D. \& Mallery, P. (2003). SPSS for Windows step by step: A simple guide and reference. 11.0 update (4th ed.). Boston: Allyn \& Bacon.

Gratz, K. L. \& Roemer, L. (2004). Multidimensional assessment of emotion regulation and dysregulation: Development, factor structure, and initial validation of the difficulties in emotion regulation scale. Journal of Psychopathology and Behavioral Assessment, 26(1), 41-54. https://doi.org/ 10.1023/B:JOBA.0000007455.08539.94

Gross, J. J. (1998). The emerging field of emotion regulation: An integrative review. Review of General Psychology, 2(3), 271-299. https://doi.org/10.1037/1089-2680.2.3.271

Gross, J. J. (2002). Emotion regulation: Affective, cognitive, and social consequences. Psychophysiology, 39(3), 281-291. https://doi.org/10.1017/S0048577201393198

Gross, J. J. \& John, O. P. (2003). Individual differences in two emotion regulation processes: implications for affect, relationships, and well-being. Journal of Personality and Social Psychology, 85(2), 348-362. https://doi.org/10.1037/0022-3514.85.2.348

Gross, J. J. \& Levenson, R. W. (1993). Emotional suppression: physiology, self-report, and expressive behavior. Journal of Personality and Social Psychology, 64(6), 970-986. https://doi.org/10.1037/00223514.64.6.970

Gross, J. J. \& Muñoz, R. F. (1995). Emotion regulation and mental health. Clinical Psychology: Science and Practice, 2(2), 151-164. https://doi.org/10.1111/j.1468-2850.1995.tb00036.x

Gross, M. N., Zalazar Jaime, M. F., Piccolo, N. V., \& Cupani, M. (2012). Nuevos estudios de validación del cuestionario de personalidad IPIP-FFM. Trabajo presentado en el X Congreso Latinoamericano de Sociedades de Estadística, Córdoba, Argentina.

Hair, J. F., Black, W. C., Babin, B. J., \& Anderson, R. E. (2010). Multivariate data analysis (7th ed.). Nueva York: Pearson College Division. https://doi.org/10.1016/j.jmva.2009.12.014

Hair, J. F., Black, W. C., Babin, B. J., Anderson, R. E., \& Tatham, R. L. (2006). Multivariate Data Analysis. Vol 6. Nueva York: Pearson Prentice Hall.

Hogan, T. (2004). Pruebas Psicológicas. El Manual Moderno. México D.F.: Mc Graw Hill

Hoyle, R. H. (2012). Handbook of structural equation modeling. Nueva York: Guilford Press.

Hu, L. T., Bentler, P. M., \& Kano, Y. (1992). Can test statistics in covariance structure analysis be trusted? Psychological bulletin, 112(2), 351-362. https://doi.org/10.1037/0033-2909.112.2.351

Hyrkäs, K., Appelqvist-Schmidlechner, K., \& Oksa, L. (2003). Validating an instrument for clinical supervision using an expert panel. International Journal of Nursing Studies, 40(6), 619-625. https://doi.org/10.1016/S0020-7489(03)00036-1

Kline, R. B. (2010). Principles and Practice of Structural Equation Modeling (3rd ed.). Nueva York: Guilford Press.

Koole, S. L. (2009). The psychology of emotion regulation: An integrative review. Cognition and Emotion, 23(1), 4-41. https://doi.org/10.1080/02699930802619031

Linehan M. M. (1993). Cognitive-Behavioral Treatment of Borderline Personality Disorder. Nueva York: Guilford Press.

Manzano Patiño, A., \& Zamora Muñoz, S. (2009). Sistema de ecuaciones estructurales: una herramienta de investigación: Cuaderno técnico. México D.F.: Centro Nacional de Evaluación para la Educación Superior.

McDonald, R. P. (1999). Test theory: A unified treatment. New Jersey: Lawrence Erlbaum Associates.

Medrano, L. A., Moretti, L., Ortiz, Á., \& Pereno, G. (2013). Validación del cuestionario de regulación emocional cognitiva en universitarios de Córdoba, Argentina. Psykhe, 22(1), 83-96. https://doi.org/10.7764/psykhe.22.1.473

Medrano, L. A. \& Trógolo, M. (2014). Validación de la Escala de Dificultades en la Regulación Emocional en la población Universitaria de Córdoba, Argentina. Universitas Psychologica, 13(4), 15-26. https://doi.org/10.11144/Javeriana.UPSY13-4.vedr

Mennin, D. S. (2006). Emotion Regulation Therapy: An Integrative Approach to Treatment-Resistant Anxiety Disorders. Journal of Contemporary Psychotherapy, 36(2), 95-105. https://doi.org/10.1007/s10879006-9012-2

Muñiz, J., Elosua, P., \& Hambleton, R. K. (2013). Directrices para la traducción y adaptación de los tests: segunda edición. Psicothema, 25(2), 151-157. 
Nunnally, J. C. \& Bernstein, I. H. (1994). Psychometric theory (3rd ed.). Nueva York: McGraw Hill.

Revelle, W. (2018). Psych: Procedures for psychological, psychometric, and personality research. $R$ package version 1.8 .12

Rice, S. M., Treeby, M. S., Gersh, E., Ogrodniczuk, J. S., \& Kealy, D. (2018). The emotion regulation questionnaire: ERQ-9 factor structure and measurement invariance in Australian and Canadian community samples. Testing, Psychometrics, Methodology in Applied Psychology, 25(3), 369-377.

Sandín, B., Chorot, P., \& Valiente, R. M. (2012). Transdiagnóstico: nueva frontera en psicología clínica. $\begin{array}{lllll}\text { Revista de Psicopatología y Psicología } & \text { Clínica, } & 17(3), & 185-203 .\end{array}$ https://doi.org/10.5944/rppc.vol.17.num.3.2012.11839

Schumacker, R. E. \& Lomax, R. G. (2016). A beginner's guide to structural equation modeling. Nueva York: Routledge.

Schweizer, K. (2010). Some Guidelines Concerning the Modeling of Traits and Abilities in Test Construction. European Journal of Psychological Assessment, 26(1), 1-2. https://doi.org/10.1027/1015$5759 / \mathrm{a} 000001$

Spaapen, D. L., Waters, F., Brummer, L., Stopa, L., \& Bucks, R. S. (2014). The Emotion Regulation Questionnaire: Validation of the ERQ-9 in two community samples. Psychological Assessment, 26(1), 46-54. https://doi.org/10.1037/a0034474

Tinsley, H. E. \& Weiss, D. J. (1975). Interrater reliability and agreement of subjective judgments. Journal of Counseling Psychology, 22(4), 358-376. https://doi.org/10.1037/h0076640

Tornimbeni, S., Pérez, E., Olaz, F., de Kohan, N. C., Fernández, A., \& Cupani, M. (2008). Introducción a la psicometria. Buenos Aires: Paidós.

Voutilainen, P., \& Liukkonen, A. (1995). Senior Monitor-laadun arviointimittarin sisällön validiteetin määrittäminen. Hoitotiede, 7(1), 51-56.

Wiltink, J., Glaesmer, H., Canterino, M., Wölfling, K., Knebel, A., Kessler, H., \& Beutel, M. E. (2011). Regulation of emotions in the community: Suppression and reappraisal strategies and its psychometric properties. GMS Psycho-Social-Medicine, 8, 1-12.

World Medical Association. (2013). Declaration of Helsinki. Ethical principles for medical research involving human subjects. JAMA Network, 310(20), 2191-2194. https://doi.org/10.1001/jama.2013.281053

Recibido 18-07-2020 | Aceptado 04-12-2020

Este trabajo se encuentra bajo una Licencia Creative Commons Atribución 4.0 Internacional que permite a terceros utilizar lo publicado siempre que se dé el crédito pertinente a los autores y a Psicodebate 